\title{
Diagnostic accuracy in thermographic imaging for granulation of uninfected diabetic ulcers.
}

\author{
Rodríguez-Alonso $\mathrm{D}^{*}$, Cabrejo-Paredes $\mathrm{J}^{\star *}$ and Benites-Castillo $\mathrm{S}^{* * *}$
}

\author{
*“Innovaciones pie diabetico" medical Centre, Trujillo, Peru, ortocentro30@gmail.com \\ ${ }^{* *}$ Postgraduate research Unit, National University of Trujillo ,Trujillo, Peru, jcabrejop@gmail.com \\ ${ }^{* * *}$ Research Institute, University of Cesar Vallejo, Trujillo, Peru, santiagob@ucv.edu.pel
}

\begin{abstract}
The aim was to determine accuracy by sensitivity (Se), specificity (Sp) of thermographic imaging test in the granulation of uninfected diabetic ulcers in medical Center. 29 diabetic type 2 with 41 uninfected diabetic ulcers were transversally design comparing granulation area by thermographic image respect to standard by pathological anatomical study. The results of uninfected diabetic ulcers were in predominance for type 2 and type A by Wagner and University of Texas classification in $70.7 \%$ and $51.2 \%$ respectively. The diagnostic test had Se in $100 \%$, Sp in $71.4 \%$ and accuracy in $90.2 \%$. The accuracy was ideal.
\end{abstract}

\section{Introduction}

The International Diabetes Federation states that "every 30 seconds a leg of a diabetic is amputated somewhere in the world and causes an economic expense five times more in those with ulcers than those who do not have it" [1].

Diabetic ulcer is a chronic continuity solution caused by factors such as peripheral neuropathy, peripheral arterial disease, precipitate deformity due to inadvertent trauma in a patient with hyperglycaemia[2].The morphological characteristics of chronic ulcers are size, shape, anatomical site, pain, exudate and bed[3].The healing phases of chronic ulcers are inflammatory, proliferative and remodelling[4].The proliferative phase is characterized by angiogenesis, collagen synthesis and wound contraction and have its microscopic correlation in the presence of blood vessel formation, extracellular matrix, chronic inflammatory infiltrate and presence of fibroblasts that are arranged in layers [5]. Biopsy of chronic ulcers can identify the etiological diagnosis and histological content [6].

A systematic review concluded that non-invasive imaging techniques such as digital photography, hyperspectral imaging, thermography, laser doppler imaging and focal microscopy have immense potential to evaluate healing of chronic ulcers in progress and severity over time [7]. Medical infrared thermography is an imaging technique that studies human thermal physiopathology as a diagnostic method that captures and records infrared electromagnetic thermal energy emitted by human body radiation and that transforms it into an electronic image signal, which is amplified to a monitor in colour tones according to magnitude[8].A narrative review states that infrared thermography has been successfully used to detect complications related to the diabetic foot because it is fast, non-contact, non-invasive and allows visualization of the temperature distribution of the foot[9].Thermographic measurements in perilesional to intralesional temperature difference from 1 to $3{ }^{\circ} \mathrm{C}$ are related to a granulation state and from $3{ }^{\circ} \mathrm{C}$ to more as an infection with respect to a clinical pattern of infectious symptoms and signs[10].

The identification of new technique in physiological evaluation of healing of diabetic ulcers, measurement of accuracy of the granulation activity with respect to histological pattern and the methodological contribution in diagnostic tests in use of thermography in diabetic ulcers justified to determine accuracy by sensitivity, specificity of the thermographic imaging test respect to histological test in the granulation of uninfected diabetic ulcers treated in a diabetic foot unit.

\section{Material and methods}

This study had a cross-sectional design of simple diagnostic tests about thermographic imaging test versus histological study test respect to granulation of diabetic ulcers. The dependent variable was presence of granulation in diabetic ulcers with qualitative measurement and independent variables were thermographic imaging test and histologic study test with qualitative measurements. The population were all diabetic foot ulcer patients evaluated in "Innovaciones pie diabetic" medical Centre. The sample size was a minimum of 41 ulcers calculated by Bugang et al methodology[11] in considering sensitivity in $100 \%$ and specificity in $66.6 \%$ (preliminary study) and prevalence of LatinAmerican study[12]. The observational unit and analysis was the granulation area of the non-infectious diabetic ulcer. The inclusion criteria were diabetic ulcers that had been submitted to instrument called "Thermalulcer", located in foot, previous preparation of bed ulcer and a minimum of one month in healthcare. The exclusion criterion were diabetic ulcers in metabolic, infection and vascular urgency.

The collection technique was called "Thermalulcer" validated by 3 experts. The methodology was to obtain clinical data and images which was performed by a family physician after training. Image analysis was performed by principal researcher, biopsy by a trained physician and histological analysis by a pathologist. 
The examination oriented to peripheral neurovascular part and ulcer was performed by "Thermalulcer" instrument mentioned above. Thermographic imaging was obtained from FLIR E60 camera (infrared resolution $320 \times 240$ pixels and thermal sensitivity of $<0.05^{\circ} \mathrm{C}$ ). The patient's approach was at rest 15 minutes prior to taking image, distance from ulcer to camera focus was 20 to $30 \mathrm{~cm}$ perpendicular to ulcerative surface using laser pointer under identification number, good light and ambient temperature of 20 to 25 degrees, humidity of 60 to $80 \%$ according to American Thermology Academy guidelines[13].

The analysis of ulcerative imaging was performed by FLIR Tool software where were identified on intralesional and outlying areas with white-red (hottest) and blue-black (coldest) and identification of granulation was identified by calculation of outlying thermal average minus value of a hottest intralesional point being between 1 and $3{ }^{\circ} \mathrm{C}$ according to Chanmugam et al[14], Baharara et al[15], and Fierheller et al[10]methodologies. The biopsy was guided by camera using a skin punch needle (diameter of $2 \mathrm{~mm}$ ) and the hottest point was identified by camera taking security measures to avoid bleeding or contamination. Finally, histological study was performed by conventional microscopy of sheet prepared with haematoxylin and eosin considering histological criteria according to Bassetto et al[16].

Descriptive and analytical data was using EPIDAT version 4.4 . The study was approved by an ethics committee of the Faculty of Medicine of the National University of Trujillo. Informed consent was performed on all patients and following guidelines corresponding to the World Medical Association's Declaration of Helsinki.

\section{Results}

The patients recruited and evaluated were 29 diabetics with uninfected ulcers. Predominant characteristics were male gender with $62.1 \%$, average age was 60.83 years, these patients were type 2 diabetics in $100 \%$, diabetes time of more than 5 years ago was $62.1 \%$, oral diabetes treatment with $93.1 \%$, regular treatment was $37.9 \%$ and presence of amputation in $37.9 \%$. Ulcer was unique in $51.21 \%$ (21 patients), two ulcers in $19.51 \%$ (4 patients) and three ulcers in $29.26 \%$ (4 patients), peripheral neuropathy and peripheral arterial disease were presented in $75.9 \%(22$ patients) and $41.4 \%$ (12 patients) respectively .

Ulcerative exploration of 41 diabetic ulcers had an average time of illness, conventional and adjuvant treatment in 2.11 months, $51.2 \%$ and $48.8 \%$ respectively. Topographic location for plantar, dorsal and both was presented in $5.2 \%, 46.3 \%$ and $2.4 \%$ respectively. Ulcerative dimensions were $2.98 \times 2.33 \mathrm{~cm}$ average length and width respectively, affected side and affected area was the right and front foot respectively with $68.3 \%$ each one. The classification of diabetic ulcers according to Wagner was type 1,2, 3 in $24.4 \%, 70.7 \%$ and $4.9 \%$ respectively and according to the classification of the University of Texas was type A1 to A3 and C1 to C3 in $56.1 \%$ and $43.9 \%$ respectively and more details of morphologic characteristic in table 1 . The figure 1 showed an case of thermographic imaging in diabetic ulcer which had hottest area marked with $x$ corresponding to granulation and figure 2 showed it histologic imaging .

The contingency table of diagnostic tests to identify granulation in diabetic ulcers evaluated by thermographic imaging(evaluated test) versus histologic study (god standard test) were shown in Table 2 . The table was shown absence of false negatives and 4 false positives for thermographic imaging .Sensitivity, specificity and accuracy were $100 \%(87.5$ - 100) , 71.4\%(45.4-88.3), 90.3\%(77.5 - 96.1) respectively to thermographic imaging test in 41 diabetic ulcers .

Table 1. characteristics of 41 non-infected ulcers evaluated by thermographic imaging and histologic study in 29 diabetic patients.

\begin{tabular}{|l|l|l|l|}
\hline characteristic & Parameter & $\mathrm{n}$ & $\%$ \\
\hline Morphologic & General anatomical zone & & \\
& Forefoot & 28 & 68.3 \\
& Midfoot & 6 & 14.6 \\
& Rearfoot & 7 & 17.1 \\
& Specific anatomical zone & & \\
& Hallux & 18 & 43.9 \\
& Lesser toes & 10 & 24.4 \\
& Metatarsal & 4 & 9.8 \\
& Plantar midfoot and heel & 6 & 14.6 \\
& Dorsal midfoot and rearfoot & 3 & 7.3 \\
& Depth & & \\
& Superficial & 12 & 29.3 \\
& deep not bone & 27 & 65.9 \\
& deep bone & 2 & 4.9 \\
& Predominant colour & 29 & 70.7 \\
& Red & 12 & 29.3 \\
\hline
\end{tabular}


10.21611/qirt.2020.110

$15^{\text {th }}$ Quantitative InfraRed Thermography Conference, 6 - 10 July 2020, Porto, Portugal

Table 2. thermographic imaging test to identify granulation in 41 ulcers versus standard diagnostic test in 29 diabetic patients.

\begin{tabular}{|l|c|c|c|c|c|c|}
\hline \multirow{2}{*}{ Test } & \multicolumn{2}{|l|}{ Positive histologic study } & \multicolumn{2}{l|}{ Negative histologic study } & \multicolumn{2}{l|}{ Total } \\
\cline { 2 - 8 } & $\mathrm{n}$ & $\%$ & $\mathrm{n}$ & $\%$ & $\mathrm{n}$ & $\%$ \\
\hline Positive thermographic imaging & 27 & 65.9 & 4 & 9.8 & 31 & 75.6 \\
\hline Negative thermographic imaging & 0 & 0 & 10 & 24.4 & 10 & 24.4 \\
\hline Total & 27 & 65.9 & 14 & 34.1 & 41 & 100 \\
\hline
\end{tabular}

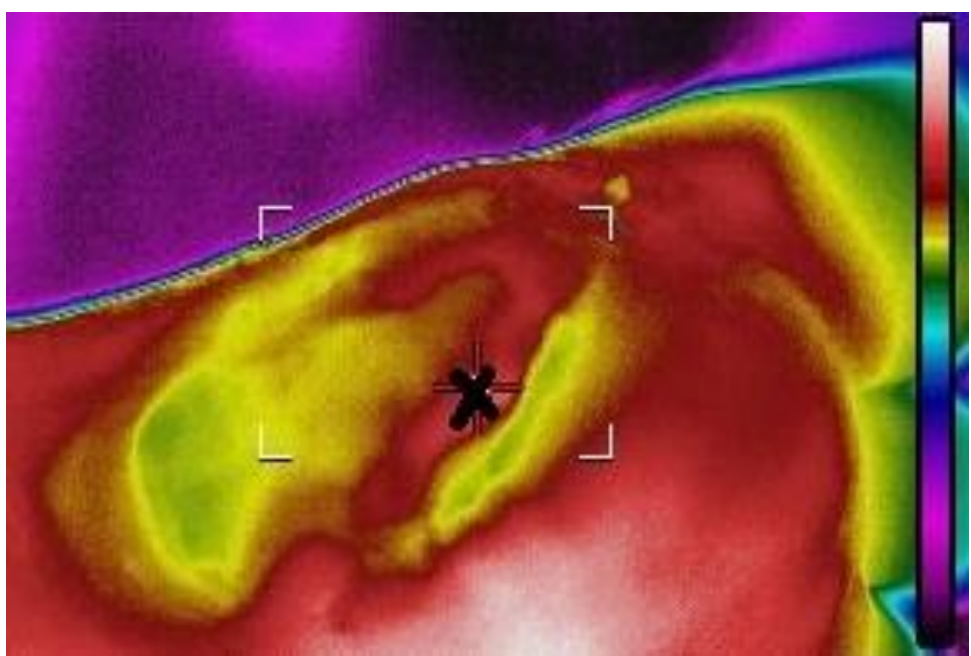

Figure 1: thermographic imaging of diabetic ulcer which showed in " $x$ " the hottest area.

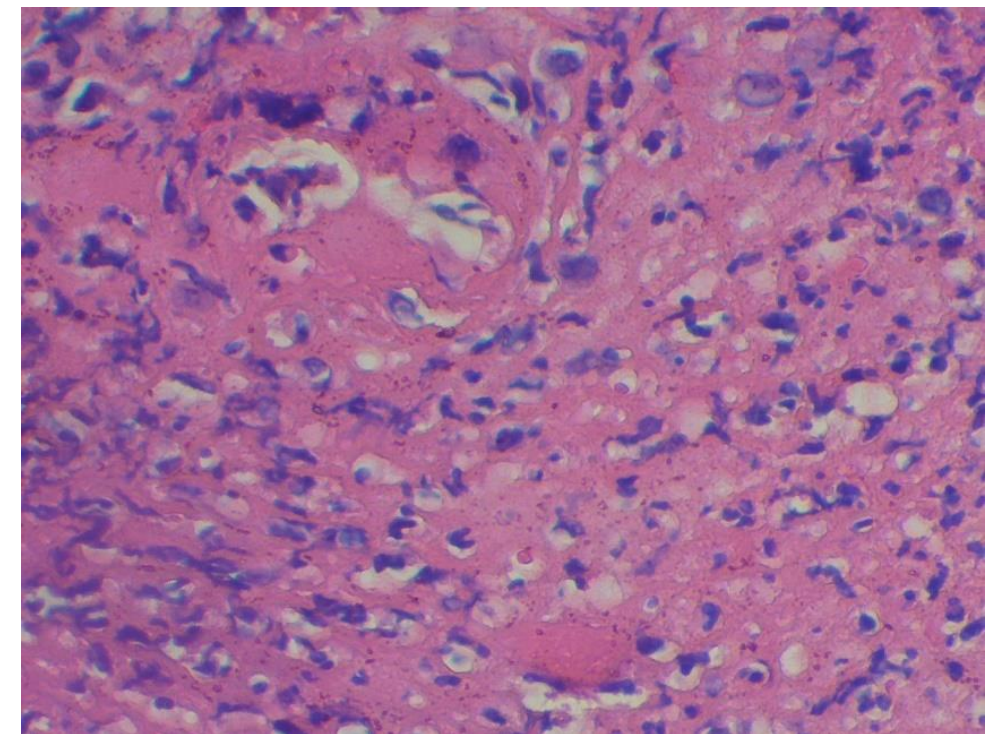

Figure 2: histologic imaging of biopsy hottest area in diabetic ulcer mentioned in figure 1. 


\section{Discussion}

Mukherjee et al [7] described multiple evaluative techniques non-invasive in chronic ulcers including thermographic imaging, but they did not conclude which were best, but emphasized that they were much better than simple observation because it provides to physician above severity, state of healing and frequency of healing in time with accuracy and precision. Therefore, methodology of this study were included within techniques accepted worldwide.

Diagnostic accuracy is the degree to which a test measures what it is supposed to measure according to Thomas et al[17].Sensitivity is possibility of having condition being truly with it and specificity is possibility of not having condition being truly absent from it[18]. Diagnostic tests are preferably used to say whether you are sick or healthy in a classical manner but can be used for presence of certain diagnostic conditions in the clinical setting according to Thompson et al[19]; in the case of this study condition was presence of granulation.

This study has found that sensitivity of thermographic imaging test was ideal to recognize granulation when the condition was present and specificity was high allowing to recognize no granulation when condition was absent. The identification of 4 cases of false positives corresponded to deep ulcers where dominant thermographic imaging corresponded anatomically to central ulcerative bed, giving positive by thermographic imaging but microscopically fibroperiosteal and fibromuscular tissue was found in histological study ; explanation of this result was that this area emits heat by their same metabolic function but they are not granulation processes and another explanation is that granulation starts from the edges of the ulcer towards centre[5].

This diagnostic test study using thermographic imaging could not be compared respect to other similar in methodology. The scientific publications has reported few studies using other assessment techniques with same subject matter such as:

-Bharara et al[13] has presented a report of a case of diabetic ulcer, where it uses the quantitative thermographic imaging and as parameter thermal index of ulcer that corresponds to division of highest temperature area between total area.

-Wang et al[20] has found a good accuracy about the hardware and software in measuring the size of different chronic ulcers including diabetic type.

-Languso et al[21] has found good reproducibility among professionals on the analysis of thermographic imaging in chronic ulcers without specifying ulcerative type.

-Chanmugam et al[14] have conducted a series of case studies describing thermographic imaging associated with infection, inflammation and granulation in chronic ulcers but they did not include diabetic types.

-Aliahmad et al[22] have performed a study to follow diabetic ulcers for four weeks looking for perilesional thermal equilibrium with respect to intralesional to identify granulation using thermographic imaging .

Physical bases of thermographic imaging described by Vollmer and Möllmann[23] are based on principles of Planck's law considering that radiation of an object (skin for this case)respect to temperature function, wavelength and emissivity can be applied to chronic ulcers and second law of thermodynamics which is related to balancing a body or zone from hot to cold until equilibrium ;this was applied in ulcerative granulation by high metabolic activity caused by angiogenesis according to Waleed et al[24]. Emissivity is a necessary parameter to take into consideration to avoid calculation errors in the image analysis process according to Keenan et al [25];this study considered the emissivity into imaging analysis .

Although the aim of this study was not only found thermographic reference points between the intralesional and perilesional areas to consider presence of granulation according to Chanmugam et al[12] and Fierheller et al[8] methodologies ; therefore, parameter such as diabetic type and dimensions of ulcers considered in this study should be considered in future studies. This study has a high impact within the clinical diagnosis because thermographic imaging can be used as a non-invasive and very accurate tool to help demonstrate physiological process of healing that will prevent inaccuracy, monitor ulcers and impact on costs of treatments.

In conclusion, this study was original in its design methodology to evaluate the diagnostic test of thermographic imaging in granulation of uninfected diabetic ulcers, finding a very high sensitivity and specificity. Therefore, these innovative non-invasive techniques are recommended in the clinical context to improve the accuracy of the diagnostic process of granulation of diabetic ulcers.

\section{REFERENCES}

[1] International Diabetes Federation.; IDF Diabetes Atlas, pp.82-95, 9th edn, Brussels, Belgium: 2017. Available at: https://www.diabetesatlas.org.

[2] Noor S., Zubair M., Ahmad J., Diabetic foot ulcer:A review on pathophysiology, classification and microbial etiology. Diabetes Metab Syndr, vol 9,pp.192-199, 2015.

[3] Hess CT., Assessing and documenting chronic wounds In: Hess CT., Wound care. pp 15-33, 7th edn, Wolters Kluwer, Philadelphia ,2013.

[4] Ghatak S., Hascall VC., Moreno-Rodriguez R., et al., Inflammation, Wound healing and fibrosis, In: Turksen K., Wound Healing: stem cells repair and restorations, basic and clinical aspects,pp. $195-209,1^{\text {st }}$ edn, John Wiley \& Sons, New Deli ,2018. 
[5] Sussman C., Skin and soft tissue anatomy healing physiology In : Sussman C., Bates-Jensen B., Wound care. pp. 17-48, $4^{\text {th }}$ edn, Wolters Kluwer,Philadelphia ,2013.

[6] Alavi A., Niakosari F., Sibbald RG., When and How to Perform a Biopsy on a Chronic Wound. Adv skin wound care, vol. 23, pp 132-40, 2010.

[7] Mukherjee R., Tewary S., Routray A., Diagnostic and Prognostic Utility of Non-Invasive Multimodal Imaging in Chronic Wound Monitoring: A Systematic Review, J Med Syst ,vol. 412, pp.46-65,2017.

[8] Brioschi M., Teixeira ML., Silva MT., Colman FM., Medical Thermography Textbook: Principles and Applications. Pp. 9, first edition, Editora e Livrari Andreoli , Sao Paolo, 2010.

[9] Hernandez-Contreras D., Peregrina-Barreto H., Rangel-Magdaleno J., Gonzalez-Bernal J., Narrative review: Diabetic foot and infrared thermography. Infrared Physics \& Technology , vol. 78, pp. 105-117, 2016.

[10] Fierheller M., Sibbald RG., A Clinical Investigation into the Relationship between Increased Periwound Skin Temperature and Local Wound Infection in Patients with Chronic Leg Ulcers, Adv skin wound care.Vol. 23,pp. 369-79, 2010.

[11] Bujang MA., Adnan TH., Requirements for Minimum Sample Size for Sensitivity and Specificity Analysis. J Clin Diagn Res ,vol. 10,pp.1-6, 2016.

[12] Carro G., Saurral R., Salvador F., Witman E., Pie diabético en pacientes internados en hospitales de Latinoamérica. Medicina (Buenos Aires),Vol.78 , pp.243-51, 2018.

[13] American Academy of Thermology., Guidelines for neuromusculoskeletal infrared thermography sympathetic skin response (SSR) studies., Pan Am J Med Thermol,Vol.2,pp.35-43,2015.

[14] Chanmugam A., Langemo D., Thomason K., Haan J., et al., Relative temperature maximum in wound infection and inflammation as compared with a control subject using long-wave infrared thermography. Adv Skin Wound Care, Vol.30, pp. 406-14, 2017.

[15] Bharara M., Schoess J., Nouvong A., Armstrong DG., Wound inflammatory index: a "proof of concept" study to assess wound healing trajectory. J Diabetes Sci Technol, Vol.4, pp. 73-9,2010.

[16] Bassetto F., Lancerotto L., Salmaso R., Pandis L., et al., Histological evolution of chronic wounds under negative pressure therapy. Journal of Plastic, Reconstructive \& Aesthetic Surgery,Vol. 65,pp.91-9, 2012.

[17] Thomas RL., Zidan MA., Slovis TL., What you need to know about statistics Part I: validity of diagnostic and screening tests. Pediatr Radiol,Vol. 54,pp.146-52, 2015.

[18] Leeflang MM., Systematic reviews and meta-analyses of diagnostic test accuracy. Clin Microbiol Infect ,Vol. 20,pp.105-13,2014

[19] Thompson M., Van den Bruel A., Diagnostic Tests Toolkit,pp.3-4,1st edn, John Wiley \& Sons, Oxford, 2012.

[20] Wang S., Anderson JA., Evans R., Woo K., et al., Point-of-care wound visioning technology: Reproducibility and accuracy of a wound measurement app. PLoS ONE, Vol. 12, pp.1-14, 2017.

[21] Langemo DK., Spahn JG., A Reliability study using a long-wave infrared thermography device to identify relative tissue temperature variations of the body surface and underlying tissue. Adv Skin Wound Care,Vol. 30,pp. 10919, 2017.

[22] Aliahmad B., Tint AN., Poosapadi-Arjunan S., Rani P., et al., Is thermal imaging a useful predictor of the healing status of diabetes-related foot ulcers? a pilot study. J Diabetes Sci Technol ,Vol.13,pp. 561-7, 2018.

[23] Vollmer M., Möllmann KP., Fundamentals of infrared thermal imaging In: Vollmer M.,Möllmann KP., Infrared thermal imaging fundamentals, research and applications ,pp.1-106, $2^{\text {nd }}$ edition, Wiley-VCH; Weinheim ,2018.

[24] Waleed HA., Imran S., Abeer YM., Muhammad HH., Assessment methods for angiogenesis and current approaches for its quantification. Indian J Pharmacol, Vol.46,pp. 251-6, 2014.

[25] Keenan E., Gethin G., Flynn L., Watterson D., O'Connor GM., Enhanced thermal imaging of wound tissue for better clinical decision making. Physiol Meas, Vol.38,pp. 1104-15, 2017. 